\title{
Magnetic field effects on low dimensional electron systems: Luttinger liquid behaviour in a Quantum Wire
}

\author{
S. Bellucci ${ }^{1}$ and P. Onorato ${ }^{1} 2$ \\ ${ }^{1}$ INFN, Laboratori Nazionali di Frascati, P.O. Box 13, 00044 Frascati, Italy. \\ ${ }^{2}$ Dipartimento di Scienze Fisiche, Università di Roma Tre, Via della Vasca Navale 84, 00146 Roma, Italy
}

(Dated: November 15, 2018)

\begin{abstract}
We discuss the effects of a strong magnetic field in Quantum Wires. We show how the presence of a magnetic field modifies the role played by electron electron interaction producing a strong reduction of the backward scattering corresponding to the Coulomb repulsion. We discuss the consequences of this and other effects of magnetic field on the Tomonaga-Luttinger liquids and especially on their power-law behaviour in all correlation functions.

The focal point is the rescaling of all the repulsive terms of the interaction between electrons with opposite momenta, due to the edge localization of the electrons and to the reduction of the length scale. Because of the same two reasons there are some interesting effects of the magnetic field concerning the backward scattering due to the presence of one impurity and the corresponding conductance. As an effect of the magnetic field we find also a spin polarization induced by a combination of electrostatic forces and the Pauli principle, quite similar to the one observed in large Quntum Dots.

PACS numbers: 73.21.Hb, 71.10.Pm,73.21.La
\end{abstract}

\section{INTRODUCTION}

In the last 20 years progresses in semiconductor device fabrication and carbon technology allowed the construction of several new devices at the nanometric scale and many novel transport phenomena have been revealed in mesoscopic low-dimensional structures.

Molecular beam epitaxy allows one to construct interesting two-dimensional devices in heterostructures between different thin semiconducting layers (a strong electric field creates a two dimensional electron gas (2DEG) at the interface) while other techniques (such as the electron beam lithography) for the deposition of metallic gates allow us to confine electrons in small devices with controllable size and contact transparency ${ }^{1}$.

Semiconductor Quantum Wires (QWs) are quasi onedimensional(1D) devices where the electron waves are in some ways analogous to electromagnetic waves in waveguides. They are made from a $2 \mathrm{DEG}$ at the interface of a $G a A s$ : AlGaAs heterojunction where a quasi one dimensional electron gas can be formed by etching the heterojunction into a wire of width, say, $1000 \AA^{1}$.

The transport in QWs is connected to three different regimes, the two ones at very low temperatures correspond to the typical single electron tunnelling (Coulomb blockade) and to Ballistic Transport (where the Landauer-Büttiker formalism applies 2.3.4 while when the correlation is strong the TomonagaLuttinger ${ }^{5.6 .7 .8}$ liquid regime dominates.

Experiments with short one-dimensional (1D) conductors (QWs, narrow ballistic channels, quantum point contacts in a 2DEG and Carbon Nanotubes) have demonstrated ${ }^{9.10}$ that their conductance is quantized in integer multiples of $2 e^{2} / h$. However, this simple step-like form for the conductance as a function of the Fermi en- ergy, occurs when the transition between the wide leads and the narrow channel is adiabatio 11 .

The ballistic transport characterizes the motion of electrons in nanometric regions in semiconductor structures at very high electric field when velocities are much higher than their equilibrium thermal velocity. We suppose that ballistic electrons are not subjected to scattering with others electrons. A general model for near-equilibrium ballistic transport is due to the Landauer ${ }^{3}$ and Büttiker ${ }^{4}$ contributions that are condensed in the so called Landauer formula. This formula expresses the conductance of a system at very low temperatures and very small bias voltages in terms of the quantum mechanical transmission coefficients. The conductance is calculated directly from the energy spectrum by relating it to the number of forward propagating electron modes at a given Fermi energy.

Electron transport in QWs attracts considerable interest also because of the fundamental importance of the electron-electron (e-e) interaction in 1D systems: the e-e interaction in a 1D system is expected to lead to the formation of a Tomonaga-Luttinger (TL) liquid with properties very different from those of the non-interacting Fermi gas $8.12,13$.

In the TL model two types of fermions right movers and left movers are coupled by an interaction of strength g. The interaction between electrons in one-dimensional metals gives several singular properties not present in conventional (Fermi liquid) metals: (i) a continuous momentum distribution function $n(k)$, varying with $k$ as $\left|k-k_{F}\right|^{\alpha}$ with an interaction-dependent exponent $\alpha$, consequence of the lack of fermionic quasi-particles; (ii) a similar power-law behaviour in all correlation functions; (iii) charge-spin separation: the elementary excitations of a TL liquid are not quasi-particles, with charge $e$ and 
spin $1 / 2$ but collective charge and spin density fluctuations with bosonic character, i.e. so-called spinons and holons. These spin and charge excitations propagate with different velocities which lead to the separation of spin and charge.

The interest in TL liquids increased in recent years because of several new physical realizations, including quantum Hall edge systems.14.15, carbon nanotubes ${ }^{16.17}$, and semiconductor QWs ${ }^{18,19}$ in particular. Most of these experiments concentrated on the power-law behavior of the electron tunneling.

The low-energy behavior of Luttinger liquids is dramatically affected by impurities and Carbon nanotubes enable experimentalists nowadays to analyze systems with a single impurity in an otherwise perfectly pure onedimensional metal.

Because of the electron-electron interaction the backscattering amplitude generated by the impurity grows at low energy scales, so that the impurity acts as an increasingly high barrier ${ }^{20}$. A power-law singularity of the $2 k_{F}$ density response function in a Luttinger liquid can confirm this behavior. As a consequence, universal scaling behavior is expected in the low energy limit, with exponents depending only on bulk parameters of the system, rather than on the impurity strength.

A Luttinger liquid in strong magnetic field can be dramatically modified by spin effects. The presence of a strong magnetic field acting on a many electron system can induce a spin polarization. In semiconducting devices this polarization is not an effect of Zeeman coupling but it could be a result of the Coulomb exchange or a consequence of a transverse electric field always present at the interface (Rashba effect) 21.22.23. In fact the Spin Orbit (SO) coupling due to the electric field in the $z$ direction is stronger than the Zeeman term of interaction connected to a magnetic field acting on the system because of the strong reduction of the effective electron mass $\left(m^{*}=0.068 m_{0}\right)$. The Zeeman spin splitting term is $g^{*} \mu_{B} B$ where $g^{*}$ is the effective magnetic factor for electrons in this geometry (very low in GaAs) and $\mu_{B}$ is the Bohr magneton with the bare mass. So the mass renormalization reduces by a factor 10 the SO coupling and by a factor 100 the Zeeman spin splitting.

The spin behavior of an electron liquid under the effect of a strong magnetic field was accurately studied in a different nanometric semiconducting device, the Quantum Dots (QDs), which are small structures (typically less than $1 \mu \mathrm{m}$ in diameter) containing from one to a few thousand electrons. In QDs the electronic spins align when a strong magnetic field is present and it is known that magnetism occurs not because of direct magnetic forces (Zeeman coupling or SO coupling), but rather because of a combination of electrostatic forces and the Pauli principle as was proven in the last decade in several experiments 25 .
In this paper we present a study of the magnetic field dependence of Luttinger liquids in QWs. In section II we introduce the model for a QW under the action of a magnetic field. In section III we discuss the effect of the magnetic field on the kinetic and interaction coefficients as well as on the derived parameters. In particular we analyze the effects of the magnetic field on the critical coefficient $\alpha$ which characterizes the transport of the Wire because it determines the power law behaviour of the Density of the States. In section III we also discuss the effects of one impurity by analyzing the effects of magnetic field on the conductance. In section IV we analyze how a spin polarization could be observed in QWs in analogy with what happens in large QDs; we also discuss the effects on Luttinger liquid behaviour due to the spin polarization which implies the transition from a spinful Luttinger liquid to a spinless one.

\section{HAMILTONIAN AND MICROSCOPIC APPROACH}

A QW is usually defined by a parabolic confining potential along one of the directions in the plane 22,23,26: $V(x)=\frac{m_{e}}{2} \omega_{d}^{2} x^{2}$. We also consider a uniform magnetic field $B$ along the $\hat{z}$ direction which allows a free choice in the gauge determination. We choose the gauge so that the system has a symmetry along the $\hat{y}$ direction, $\mathbf{A}=(0, B x, 0)$, so that the single particle Hamiltonian is

$$
H=m_{e} \frac{\mathbf{v}^{2}}{2}+\frac{m_{e} \omega_{d}^{2}}{2} x^{2}
$$

where $m_{e} v_{y}=p_{y}-e B x /\left(m_{e} c\right)$ and $m_{e} v_{x}=p_{x}$.

In order to solve the Hamiltonian for QWs we introduce the cyclotron frequency $\omega_{c}=\frac{e B}{m c}$ and the total frequency $\omega_{T}=\sqrt{\omega_{d}^{2}+\omega_{c}^{2}}$ and point out that $p_{y}=$ $v_{y}+e B x /\left(m_{e} c\right)$ commutes with the Hamiltonian

$$
H=\frac{\omega_{d}^{2}}{\omega_{T}^{2}} \frac{p_{y}^{2}}{2 m_{e}}+\frac{p_{x}^{2}}{2 m_{e}}+\frac{m \omega_{T}^{2}}{2}\left(x-x_{0}\right)^{2},
$$

where $x_{0}=\frac{\omega_{c} p_{y}}{\omega_{T}^{2} m_{e}}$. The classical Hamilton equations give us the orbital motion in the special case of vanishing $\dot{x}(0)$

$$
\begin{aligned}
& x(t)=x_{0}+R \cos \left(\omega_{T} t\right) \\
& y(t)=v_{d} t-\frac{\omega_{c}}{\omega_{T}} R \sin \left(\omega_{T} t\right)+y(0)
\end{aligned}
$$

where the drift velocity is $v_{d}=\frac{\omega_{d}^{2} p_{y}}{\omega_{T}^{2} m_{e}}$. We obtain $p_{y}=$ $m_{e}\left(\dot{y}(0)+\omega_{c} x(0)\right), y(0)=0$ and $R=x(0)-x_{0}$ from the boundary conditions. The two different motions along the Wire are localized on the two different edges, as we can argue from the introduction of $\pm p_{y} \rightarrow \pm v_{d}$. These are also known in quantum mechanics as edge states ${ }^{26}$. 
The quantum mechanics approach to the single particle Hamiltonian in eq.(2) gives two term: a quantized harmonic oscillator and a quadratic free particle-like dispersion. This kind of factorization does not reflect itself in the separation of the motion along each axis because the shift in the center of oscillations along $x$ depends on the momentum $k_{y}$. Therefore each electron in the system has a definite single particle wave function

$$
\begin{gathered}
\varphi_{n, k_{y}}(x, y)=u_{n}\left(x-x_{0}\left(k_{y}\right)\right) \frac{e^{i k_{y} y}}{\sqrt{2 \pi L_{y}}}, \\
u_{n}\left(x-\gamma_{\omega} k\right)=\frac{1}{\sigma_{\omega} \sqrt{\pi}} e^{-\frac{\left(x-\gamma_{\omega} k\right)^{2}}{2 \sigma_{\omega}^{2}}} h_{n}\left(x-\gamma_{\omega} k\right) .
\end{gathered}
$$

Here $h_{n}\left(x-x_{0}\left(k_{y}\right)\right)$ is the $n$ Hermite polynomial shifted by $x_{0}\left(k_{y}\right)=\gamma_{\omega} k_{y}$ where $\gamma_{\omega}=\frac{\omega_{c} \hbar}{\omega_{T}^{2} m_{e}}$ and $\sigma_{\omega}=\sqrt{\frac{\hbar}{m_{e} \omega_{T}}}$. In what follows we assume $\sigma_{0}=\sqrt{\frac{\hbar}{m_{e} \omega_{d}}}$ (corresponding to zero magnetic field) as the characteristic length of the system when the magnetic field vanishes.

Now we are ready to give a simple expression of the free electron energy depending on both the $y$ momentum $k$ and the chosen subband $n$ :

$$
\varepsilon_{n, k}=\frac{\omega_{d}^{2}}{2 m_{e} \omega_{T}^{2}} \hbar^{2} k^{2}+\hbar \omega_{T}^{2}\left(n+\frac{1}{2}\right)
$$

Below we limit ourselves to electrons in a single channel $(n=0)$, where $N$ electrons occupy the lowest energy levels. We obtain the value of the Fermi wavevector as $k_{F}=\frac{N}{4} \delta k$ where $\delta k=\frac{2 \pi}{L}$.

The typical Luttinger model starts from the hypothesis that the Fermi surface consists of two Fermi points, in the neighborhood of which the dispersion curve can be approximated by straight lines with equations

$$
\varepsilon_{k} \approx v_{F}\left(|k|-k_{F}\right) \equiv v_{F} \bar{k} .
$$

In our case we obtain a field-dependent free Fermi velocity

$$
v_{F}=\frac{\omega_{d}^{2}}{m_{e} \omega_{T}^{2}} \hbar k_{F} \approx \frac{\omega_{d}^{2}}{m_{e} \omega_{c}^{2}} \hbar k_{F},
$$

where the approximation is valid for very strong fields.

We introduce different operators for the electrons belonging to each branch: right going operators $c_{R, \bar{k}, s}^{\dagger}$ and left going ones $c_{L, \bar{k}, s}^{\dagger}$ for electrons with $\bar{k}>0(\bar{k}<0)$. In terms of these operators the free and interaction Hamiltonians can be written as

$$
\begin{aligned}
H_{0} & =v_{F} \sum_{\bar{k}, s} \bar{k} c_{R, \bar{k}, s}^{\dagger} c_{R, \bar{k}, s}+v_{F} \sum_{\bar{k}, s} \bar{k} c_{L, \bar{k}, s}^{\dagger} c_{L, \bar{k}, s} \\
H_{\text {int }} & =\frac{1}{L} \sum_{k, k^{\prime}, q, s, s^{\prime}}\left(V_{k, p}^{s, s^{\prime}}(q) c_{k+q, s}^{\dagger} c_{p-q, s^{\prime}}^{\dagger} c_{p, s^{\prime}} c_{k, s}\right)
\end{aligned}
$$

Here $c_{k} \equiv c_{R, k}$ if $k>0$ and $c_{k} \equiv c_{L, k}$ if $k<0$, while $V_{k, p}^{s, s^{\prime}}(q)$ is the Fourier transform of the electron electron interaction.

The scattering processes are usually classified according to the different electrons involved and the coupling strengths labelled with $g$ are often taken as constants. In fact, as discussed in detail by Solyom ${ }^{13}$, we can substitute $V_{k, p}^{s, s^{\prime}}(q)$ with 8 constants. In general we should take into account the dependence on $k, p$ and $q$, however in a model with a bandwidth cut-off, where all momenta are restricted to a small region near the Fermi points, the momentum dependence of the coupling is usually neglected.

We can write $g_{4}^{s, s^{\prime}}$ for $k$ and $p$ in the same branch and small $q$ (transferred momentum): $g_{4}^{s, s^{\prime}}$ corresponds to the Forward Scattering in the same branch. We use $g_{2}^{s, s^{\prime}}$ for the Forward Scattering involving two branches where $k$ and $p$ are opposite and $q$ is small. The Backward Scattering $\left(g_{1}^{s, s^{\prime}}\right)$ involves electrons in opposite branches with large transferred momentum (of order $2 k_{F}$ ). We do not take into account the effects of Umklapp scattering $\left(g_{3}^{s, s^{\prime}}\right)$.

The model described above, with linear branches and constant interaction in momentum space is known as TL model and corresponds to a very short range interaction (Dirac delta). The presence of a long range interaction in a 1D electron system introduces in the model an infrared divergence and is quite difficult to solve. We reported the solutions for the case of Carbon Nanotubes obtained with a Renormalization Group approach and a Dimensional Crossover in some recent papers $\frac{27}{2}$, and in the future we will apply that formalism to the case of a $\mathrm{QW}$ in the presence of magnetic field.

Below we limit ourselves to the TL model and our main results refer to the short range interaction, with the aim of giving a qualitative explanation of the effects of a strong transverse magnetic field.

\section{LUTTINGER LIQUID PARAMETERS}

\section{A. Effective parameters}

All properties of a TL liquid can be described in terms of only two effective parameters per degree of freedom which take over in 1D the role of the Landau parameters familiar from Fermi liquid theory.

In particular the low-energy properties of a homogeneous 1D electron system could be completely specified by the TL coefficients corresponding to the interaction $\left(g_{i}^{s, \sigma}\right)$ and the kinetic energy $\left(v_{F}\right)$ in the limit of ideal TL liquid.

Four TL parameters, depending on $g$ and $v_{F}$, characterize the low energy properties of interacting spinful electrons moving in one channel: the parameter $K_{\nu}$ fixes the exponents for most of the power laws and $v_{\nu}$ is the 
velocity of the long wavelength excitations: $\nu=\rho$ for the charge and $\nu=\sigma$ for the spin. The parameters 24 $K_{\rho}$ and $v_{\rho / \sigma}$ are easily obtained as functions of $g_{i}^{s, \sigma}$ and $v_{F}$ by various techniques found in textbooks 13 .

$$
\begin{aligned}
K_{\nu} & =\sqrt{\frac{\pi v_{F}+g_{4}^{\nu}-g_{2}^{\nu}}{\pi v_{F}+g_{4}^{\nu}+g_{2}^{\nu}}} \\
v_{\nu} & =\sqrt{\left[v_{F}+\frac{g_{4}^{\nu}}{\pi}\right]^{2}-\left(\frac{g_{2}^{\nu}}{\pi}\right)^{2}} \\
\alpha & =\frac{1}{2}\left[\left(v_{F}+\frac{g_{4}^{\sigma}}{\pi}\right) \frac{1}{v_{\sigma}}+\left(v_{F}+\frac{g_{4}^{\rho}}{\pi}\right) \frac{1}{v_{\rho}}-2\right]
\end{aligned}
$$

where $g_{i}^{\sigma}=\frac{1}{2}\left(g_{i}^{\|}-g_{i}^{\perp}\right)$ and $g_{i}^{\rho}=\frac{1}{2}\left(g_{i}^{\|}+g_{i}^{\perp}\right)$. Here $\alpha$ denotes the critical exponent which characterizes many properties of the transport behaviour of a 1D device (e.g. the zero bias conductance as a function of $T$, which is well described by a power law behavior $G=T^{\alpha}$ ). In this paper we discuss microscopic estimates of the values of these quantities in semiconductor QWs when a strong transverse magnetic field is present.

\section{B. Coupling Constants}

At the end of the previous section we discussed how the electron electron repulsion has a very simple and detailed representation in the TL mode113 where the $g_{i}^{s, s^{\prime}}$ coefficients contain all the information about the interaction. In this subsection we want evaluate the $g$ coefficients which characterize the Hamiltonian eq.(17). In order to do that, we have to calculate the "Fourier transform" $V_{k, k^{\prime}}^{n, n^{\prime}}\left(q, \omega_{c}\right)$ of the effective interaction starting from some models. This is our crucial problem and gives us the $g$ coefficients. In this article we limit ourselves to the one channel model $\left(n=n^{\prime}=0\right)$ and we introduce the magnetic field dependent effective potentials

$$
\begin{aligned}
U_{k, p, q}^{s,-s} & \left(y-y^{\prime}, \omega_{c}\right)=\int_{\infty}^{\infty} d x d x^{\prime} U\left(\left|\mathbf{r}-\mathbf{r}^{\prime}\right|\right) \\
& \times u_{0}\left(x-\gamma_{\omega} k\right) u_{0}\left(x-\gamma_{\omega} p\right) \\
& \times u_{0}\left(x^{\prime}-\gamma_{\omega}(k+q)\right) u_{0}\left(x^{\prime}-\gamma_{\omega}(p-q)\right) .
\end{aligned}
$$

These potentials only depend on $y-y^{\prime}$ and on the 1D fermion quantum numbers.

Now we are ready to calculate the "Fourier transform" in order to obtain $V(q)$. A central question regards the spin effects on the coupling strength. In the HartreeFock approximation the so called direct term corresponds to $q=0(V(0))$ while the exchange one corresponds to $q=p-k(V(q))$. Is evident that the electron electron repulsion is less for electrons with the same spin $\left(V_{q}^{\uparrow \uparrow}=\right.$ $V(0)-V(q))$ than for electrons with opposite spins $\left(V_{q}^{\uparrow \downarrow}=\right.$ $V(0))$.

In order to analyze the effects of the range of the interaction we introduce a function for the electron electron potential depending on a parameter $r$. This general interaction model, ranges from the very short range one to the infinity long range one and eliminates the divergence of the Coulomb repulsion

$$
\begin{aligned}
U\left(x-x^{\prime}\right. & \left., \quad y-y^{\prime}\right)=\frac{\left(g_{0} \sigma_{0}^{2} / \pi+g_{\infty} r^{2}\right)}{r^{2}} \\
& \times \exp \left[-\frac{\left(x-x^{\prime}\right)^{2}+\left(y-y^{\prime}\right)^{2}}{r^{2}}\right]
\end{aligned}
$$

where $g_{0}$ and $g_{\infty}$ are the copuling constant corresponding to the two different limits of $r(r \rightarrow 0$ delta function $U=g_{0} \sigma_{0}^{2} \delta\left(\left|\mathbf{x}-\mathbf{x}^{\prime}\right|\right)$ and constant interaction $r \rightarrow \infty$ $\left.U=g_{\infty}\right)$.

So we can calculate $V_{k, p}\left(q, \omega_{c}\right)$ by a simple integral

$$
\begin{aligned}
& V_{k, p}\left(q, \omega_{c}\right)=\frac{\left(g_{0} \sigma_{0}^{2} / \pi+g_{\infty} r^{2}\right) \sqrt{\pi}}{L_{y}\left(\sqrt{r^{2}+2 \sigma_{\omega}^{2}}\right)} \\
& \times \exp \left[-\frac{q^{2} r^{2}}{4}-\gamma_{\omega}^{2}\left(\frac{q^{2}}{2 \sigma_{\omega}^{2}}+\frac{(k-p+q)^{2}}{r^{2}+2 \sigma_{\omega}^{2}}\right)\right] .
\end{aligned}
$$

Thus we put

$$
g(r, \omega)=\frac{\left(g_{0} \sigma_{0}^{2} / \pi+g_{\infty} r^{2}\right) \sqrt{\pi}}{L_{y}\left(\sqrt{r^{2}+2 \sigma_{\omega}^{2}}\right)}
$$

Some details about the comparison between Coulomb interaction and the model of electron electron interaction in eq.(11) are given in appendix.

Now we are ready to calculate the coefficients in eq.(7).

(a) The so called forward scattering in the same branch $\left(g_{4}\right)$ involves electrons with $p \sim k$, i.e. $\delta k=|p-k| \ll k_{F}$ : for parallel spins we have $g_{4}^{\|} \approx V_{\delta k}(0)-V_{\delta k}(\delta k)$ while for orthogonal spins we just have $V(0)$

$$
V_{\delta k}(0)=g(r, \omega) \exp \left[-\gamma_{\omega}^{2}\left(\frac{\delta k^{2}}{r^{2}+2 \sigma_{\omega}^{2}}\right)\right] \approx g(r, \omega) .
$$

So we can assume $g_{4}^{\|} \approx 0$ and $g_{4}^{\perp} \approx g(r, \omega)$.

(b) The forward scattering between opposite branches, which is usually called $g_{2}$ and corresponds to $p \sim$ $-k_{F}, k \sim k_{F} \rightarrow \delta k \approx 2 k_{F}$, where a small momentum is transferred $q \sim 0$, gives as a first approximation

$$
g_{2}^{\|}=g_{2}^{\perp} \approx V_{2 k_{F}}(0)=g(r, \omega) \exp \left[-\gamma_{\omega}^{2}\left(\frac{4 k_{F}^{2}}{r^{2}+2 \sigma_{\omega}^{2}}\right)\right] .
$$

(c) For the backward scattering $g_{1}, p \sim-k_{F}, k \sim$ $k_{F} \rightarrow \delta k \approx 2 k_{F}$ with a large momentum transferred $q \approx 2 k_{F}$, we have

$$
V_{\delta k}(0)=g(r, \omega) \exp \left[-4 k_{F}^{2}\left(\frac{r^{2}}{4}+\frac{\gamma_{\omega}^{2}}{2 \sigma_{\omega}^{2}}+\frac{4 \gamma_{\omega}^{2}}{r^{2}+2 \sigma_{\omega}^{2}}\right)\right] .
$$

Now we can discuss the effects of a magnetic field on the interaction terms and the kinetic coefficient. 

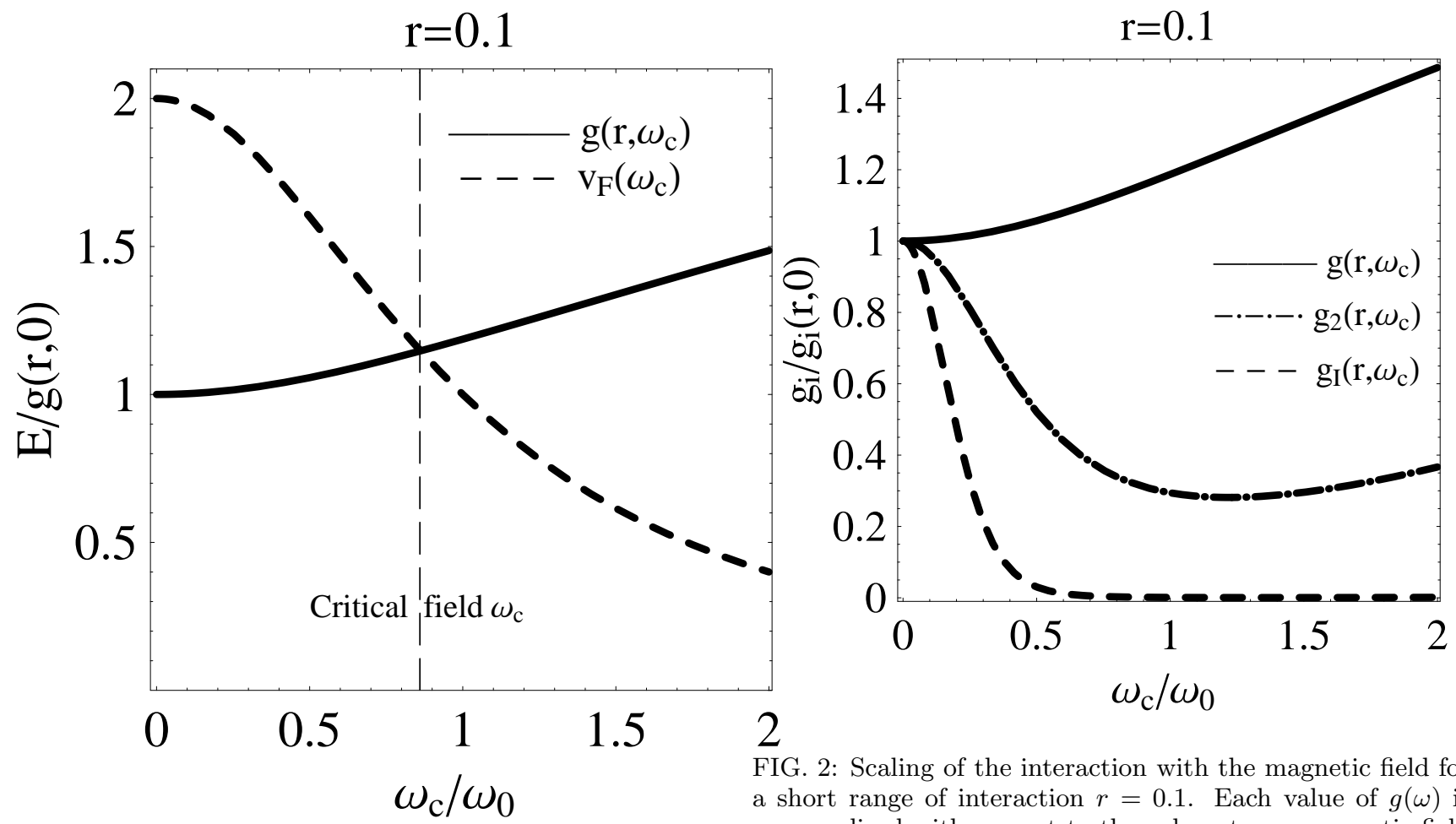

FIG. 1: Graphic calculation of the critical field: the spin transition is due to the simultaneous reduction of the Fermi velocity and increasing of the electron electron repulsion. In the y-axis we report the energy $E$ in units of $g(r, 0)$, for a starting value $v_{F}=2 g(r, 0)$.

I)A strong reduction of the Fermi velocity when the magnetic field increases is very clear, as displayed in Fig.(1) where $v_{F}\left(\omega_{C}\right)$ is reported.

II)The forward scattering between electrons in the same branch increases with the magnetic field as shown in Fig.(2) $\left(g_{4} \propto g\left(r, \omega_{c}\right)\right)$. We can also observe that the long range component of this interaction is less affected by the growing field Fig.(3).

III)The backscattering $\left(g_{1}\right)$ is strongly reduced by the magnetic field and it gives a smaller contribution to the physics of the system when the magnetic field increases. Also in this case the effect is smoothed if the interaction has long range but is however strong.

IV)The forward scattering $\left(g_{2}\right)$ between opposite branches has a strong reduction if we consider the short range component while the effect is not so clear if we take into account long range interactions.

The dependence of the coupling constants $g_{i}$ on the magnetic field shows a competitive effect of the current localization against the reduction of the characteristic length $\sigma_{\omega}$.

The localization of the electrons on the opposite edges of the wire is responsible for the strong reduction of the two constant $g_{2}$ and $g_{1}$, especially when we consider a short range interaction. The localization is clearly seen in eq.(21), where we introduced $x_{0}$ as a function of the

FIG. 2: Scaling of the interaction with the magnetic field for a short range of interaction $r=0.1$. Each value of $g(\omega)$ is renormalized with respect to the value at zero magnetic field $\left(\omega_{c}=0\right)$.

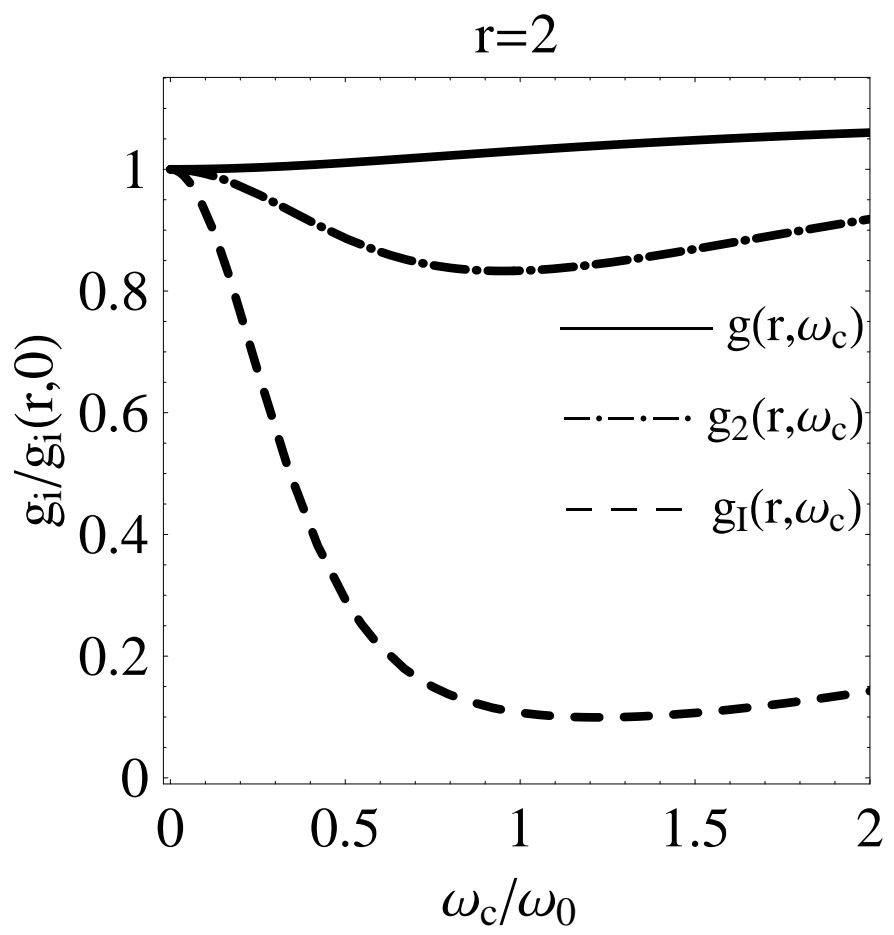

FIG. 3: Scaling of the interaction with the magnetic field for a long range of interaction $r=2$. Each value of $g(\omega)$ is renormalized with respect to the value at zero magnetic field $\left(\omega_{c}=0\right)$. 


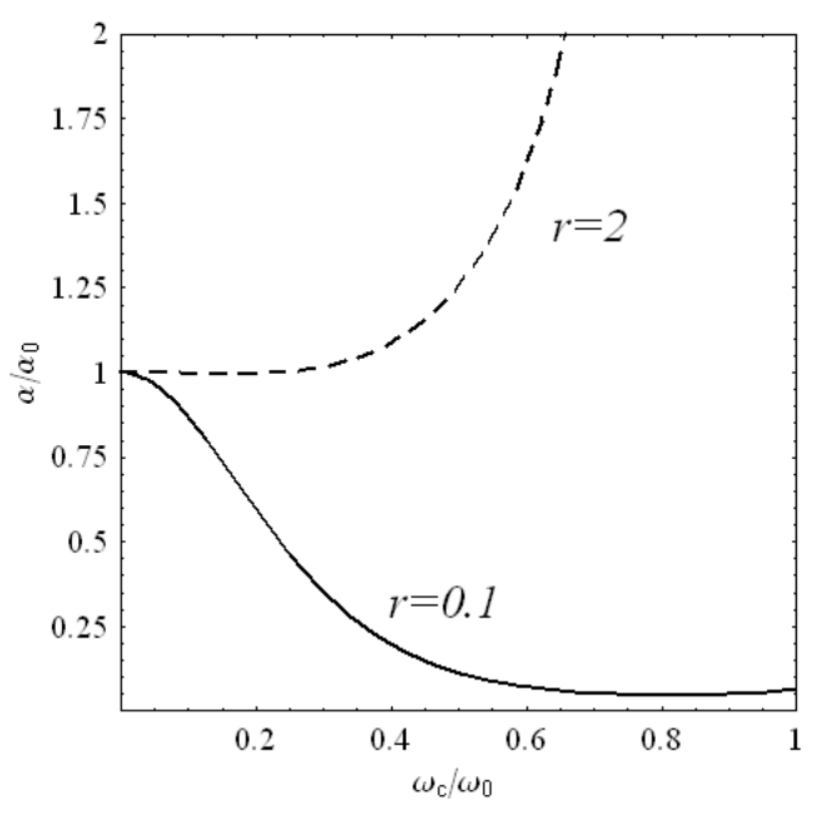

FIG. 4: The critical exponent calculated following textbooks in the limit of exactly solvable model: the range of interaction determines either a vanishing or a divergent $\alpha$.

momentum, i.e. of the drift velocity. So we can conclude that electrons with opposite drift velocities are localized in the opposite edges and a reduction in the interaction has to be observed, due to the distance between the opposite currents. Obviously this discussion is not valid if the range of the interaction is not finite.

The effects of the localization on the coupling constants are partially mitigated by the reduction of the length scale $\sigma_{\omega}$ due to the growth of the magnetic field. The typical length of the system reduces with the magnetic field and increases the effective charge density of the electron liquid. As we know, all coupling constants depend on $g\left(r, \omega_{c}\right)$ and follow its behavior when the magnetic field increases. However, just the interaction between electrons in the same branch is really enhanced by the magnetic field if we take in account the localization.

Because of the discussed failure of the TL model for a long range interaction, in order to obtain the following results we limit ourselves to the small range $(r)$ case.

\section{The critical exponent $\alpha$}

Because of our knowledge of the coupling constants we are ready to approach the problem of calculating the critical exponent $\alpha$ which characterizes the transport properties of the 1D electron systems. In fact, from the transport measurements it is possible to evaluate the Tunnel Density of States with its typical power law dependence. Usually the backscattering effect is not included in the models ${ }^{27}$ or is taken in to account as a perturbation ${ }^{8}$,

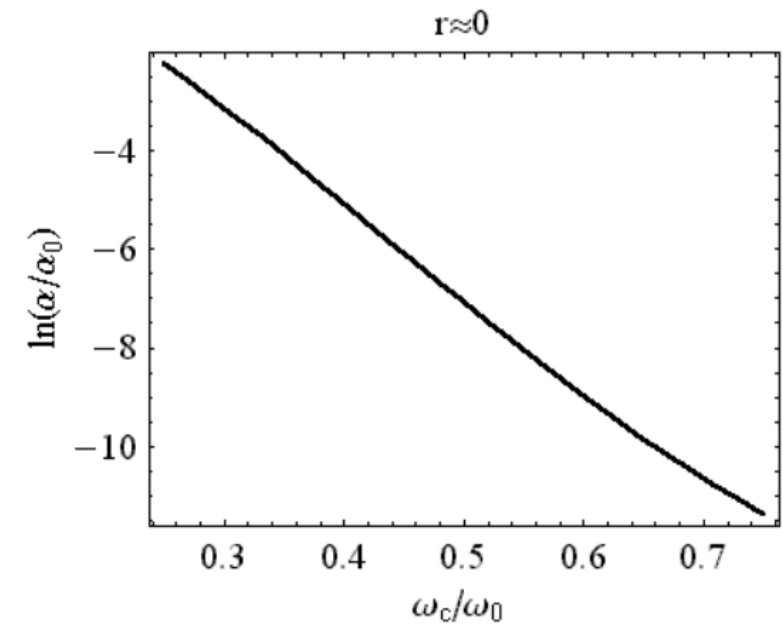

FIG. 5: The critical exponent calculated the intermediate regime, where $1 / 4<\omega_{c} / \omega_{d}<3 / 4$, the log-log plot shows the agreement with the exponential behaviour $\alpha \approx \alpha_{0} e^{-\eta \frac{\omega_{c}}{\omega_{d}}}$

so that here we do not consider $g_{1}$ more. The role of $g_{2}$ is very crucial in order to determine the critical exponent $\alpha^{12}$ which characterizes the linear temperature dependence of the resistance above a crossover temperature $T_{c}$ in a TL liquid. When $g_{2}$ vanishes the so called chiral Luttinger liquid with also $\alpha$ null appears (in that case the spin-charge separation of excitation is present but no correlation characterizes the Ground State).

The dependence of $\alpha$ on the magnetic field is our main prediction and strongly depends on the effective range of interaction. In the calculation we introduced the fielddependent coefficients in eq. (10) ${ }^{13}$. In Fig.(4) we show the strong suppression of $\alpha$ when the magnetic field increases for a small range potential. Results were obtained for values of the magnetic field $\omega_{c}<\omega_{d}$ because as we show in the next section the behaviour of the TL liquid is strongly modified in the high magnetic field regime. So we can discuss the behaviour of the critical exponent just in the weak magnetic field limit and in an intermediate one for a very short range interaction. For a very low magnetic field we can calculate

$$
\alpha=\alpha_{0}-\alpha_{0}\left(\frac{\frac{A}{4}-2 \pi v_{F}^{0}}{\left(A+2 \pi v_{F}^{0}\right) \omega_{d}^{2}}-\frac{\frac{1}{4}-\frac{4 \hbar k_{F}^{2}}{m_{e} \omega_{d}}}{\omega_{d}^{2}}\right) \omega_{c}^{2}
$$

where $A=\frac{g_{0} \sigma_{0}}{\sqrt{\pi} L_{y}}$ and $v_{F}^{0}=\frac{\hbar k_{F}}{m_{e}}$. In the intermediate regime, where $1 / 4<\omega_{c} / \omega_{d}<3 / 4$, the critical exponent reduces as follows

$$
\alpha \approx \alpha_{0} e^{-\eta \frac{\omega_{c}}{\omega_{d}}}
$$

where the constant $\eta$ is a quite complicated function depending on $A, v_{F}^{0}$ and $k_{F}$. This behaviour is clearly seen in Fig.(5). Now we can answer the question, how the magnetic field alters the Density of States exponents in the limit of short range interaction: the attenuation 
of the forward scattering between opposite branches due to the localization of the edge states is responsible for the reduction of the critical exponent. This effect dominates and characterizes the TL liquid below a value of the magnetic field where the spin polarization crossover takes place. At higher fields, as we discuss in the next section, the spin polarization causes a further reduction of $\alpha$.

In Fig.(4) we also show the critical exponent calculated for a long range interaction by using the TL model. As we discussed at the end of sec. II, the TL model fails when it is applied to the long range interaction because it intrinsically refers to a short range interaction. The divergent behaviour of the exponent when the magnetic field increases confirms the failure of the model in treating the long range interaction.

\section{Impurity and Backward scattering}

Now we want to shortly discuss how the magnetic field acts on the TL liquid when also an impurity is present in the wire. We do not give details about calculations discussed in refs. $(15,20.28)$ where the problem is mapped onto an effective field theory using bosonization and then approached by using a Renormalization Group analysis. The usual calculations start from a TL model with a scattering potential at the middle point of the wire $(x=0$ and $y=0)$ in which only forward scattering is included as electron electron interaction. Two opposite limits are usually considered: the weak potential limit and the strong potential or weak tunneling limit. The calculation usually starts from the transmission probability obtained for non-interacting electrons with a Dirac delta model for the impurity $\left(V(y)=V_{0} \delta(y)\right)$ with

$$
|t|^{2} \approx \frac{1}{1+\left(\frac{V_{0}}{\hbar v_{F}}\right)^{2}}
$$

As discussed by Kane and Fisher ${ }^{20}$ the Born approximation can be used in the small barrier limit for noninteracting electrons. This hypothesis allows us an easy calculation of the term which plays a central role in the scattering: $V\left(2 k_{F}\right)$ i.e. the Fourier transform of the potential at $2 k_{F}$. In fact ${ }^{29}$ the terms which represent scattering with momentum transfer $q \ll 2 k_{F}$ do not affect the conductance in any noticeable way, because they do not transfer particles between $k_{F}$ and $-k_{F}$. On the other hand, the terms which represent scattering with $|q| \simeq 2 k_{F}$ are expected to affect the conductance, because they change the direction of propagation of the particles. So we introduce a potential in order to describe the impurity localized at the center of the wire

$$
V(x, y)=U_{0} \frac{\sigma_{0}}{R_{0}} e^{\frac{x^{2}+y^{2}}{R_{0}^{2}}}
$$

where $R_{0}$ and $U_{0}$ represent, respectively, the range and the strength of the impurity potential.

$$
V\left(2 k_{F}\right)=\frac{\sqrt{2} V_{0} R_{0} e^{-k_{F}^{2}}\left(2 R_{0}^{2}+\frac{\gamma_{\omega}^{2}}{\sigma_{\omega}^{2}}\right)}{\sqrt{\frac{1}{2 R_{0}^{2}}+\frac{1}{\sigma_{\omega}^{2}}} \sigma_{\omega}^{2}}
$$

The strong reduction of the electron backward scattering due to the impurity depends on the magnetic field and is clearly due to the discussed localization of the edge states. This allows us to consider the weak potential limit, in order to proceed to the Renormalization Group analysis. The RG equation for $V=V\left(2 k_{F}\right)$ can be found as follows:

$$
\frac{d V}{d \ell}=\left(1-\frac{1}{2}\left(K_{\rho}+K_{\sigma}\right)\right) V=\left(1-g_{K}\right) V
$$

where $E=E_{0} e^{-\ell}$ is the renormalized cut-off and $E_{0}$ is the original one. In absence of magnetic field we can conclude that in our case, where $g_{K}<1$ corresponding to repulsive electron electron interaction, $V(\ell)$ scales to infinity. Thus at very low temperature $(T=0)$ we have a perfect reflection. However we can write a formula for the weak potential limit which gives the conductance as a function of $V_{0}, g_{K}$ and the temperature $T$ if the temperature is $T \gg 0$

$$
G_{W L}=\frac{e^{2}}{h}\left(1-c_{0} V_{0}^{2} T^{2 g_{K}-2}+\ldots\right)
$$

where ... represent higher orders in $V$ and $T$ as in ref ${ }^{15}$. As we know from the previous discussion the magnetic field acts on eq. (13) by modifying both $g_{k}$ and $V_{0}$. The formula eq. 113) rescales the conductance to 0 (total reflection) for $g_{K}<1$ and temperature below a threshold temperature. In the limit of validity of the eq. (13) we define the threshold temperature as the one for which the conductance vanishes

$$
T_{s} \approx\left(c_{0} V_{0}^{2}\right)^{-\frac{1}{2 g_{K}-2}}
$$

As we show in Fig.(6) we obtain a strong reduction of the value of $T_{s}$, due to the reduction of the single particle backscattering and to the rescaling of $g_{K}$ which approaches the value 1 corresponding to the marginal case of free electrons.

\section{MAGNETIC INDUCED PHASE TRANSITION: THE SPIN POLARIZATION}

In this section we want to discuss what happens at very strong magnetic field when a spin transition takes place in a low dimensional electron liquid.

The spin behaviour of an electron liquid in the presence of a growing transverse magnetic field was studied with some details in QDs. In 1996 Klein et al $\$ 30.31 .32$ measured the position of the conductance peaks as a function of the magnetic field in a large QD in the Coulomb Blockade 


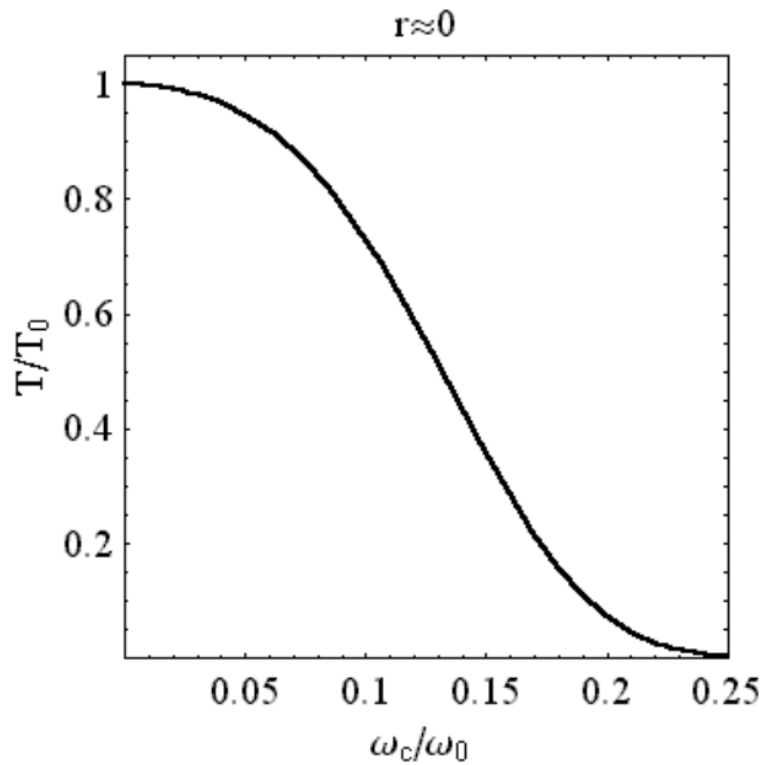

FIG. 6: The strong reduction in the value of the threshold temperature $T$, due to the reduction of the single particle backscattering and to the rescaling of $g_{K}$. The values are related to the value $T_{0}$, corresponding to the threshold temperature at zero magnetic field.

regime ${ }^{33}$. The positions of the peaks, due to a single electron tunneling, allowed them to measure the ground state (GS) energy of a many electron QD. The growth of the magnetic field yields a crossing between energy levels so that also the GS has a different spin polarization. From the measurements Klein et al. could deduce that when the magnetic field is above a threshold value, the spins flip one by one and the orbital momentum increases.

This phenomenon has a quite general explanation in the Hartree-Fock approximation and gives a very interesting phases succession by increasing magnetic field. In general we can calculate the spin transition field corresponding to the first spin flip in the dot, and a second "critical field" corresponding to the flip of the last spin.

Below we show that the magnetic field induced spin polarization takes place also in QWs and discuss the theoretical explanation in the general case. The physical mechanism which induces the transitions is very simple: the kinetic energy, proportional to the Fermi velocity, is strongly reduced by the magnetic field while the electron electron repulsion is strongly enhanced by the growing field, especially the repulsion between electrons with opposite spins (this is due to the Hund's rule).

For any model with constant interaction we can find a general condition for the spin flip and we obtain that the electron spins flip all at the same critical field. The discrepancy between the observed data and the prediction of this model will be better discussed in a following ar-
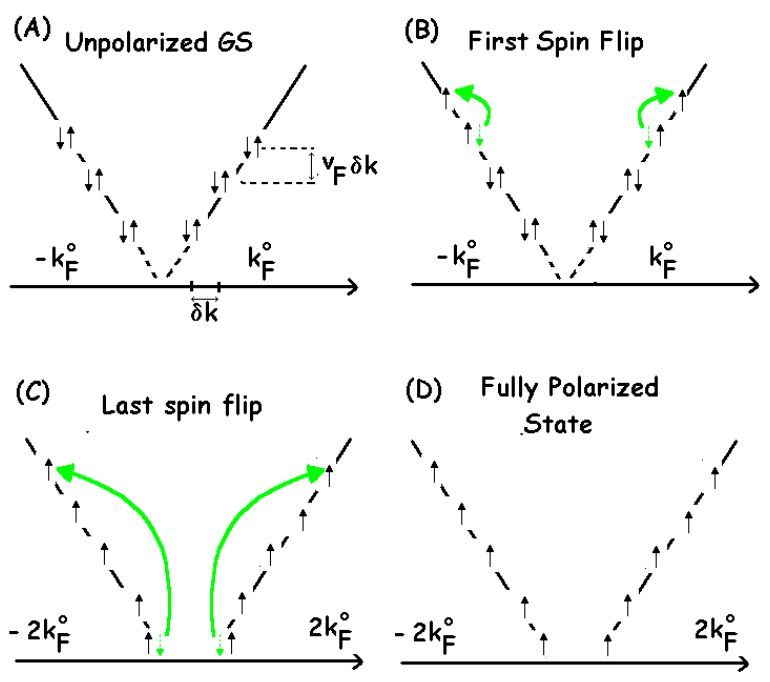

FIG. 7: A) Unpolarized state with each state doubly occupied. B) Electrons at the Fermi surface flip their spins by jumping from the $k_{F}= \pm N \delta k / 4$ doubly occupied to the nearest empty levels $\pm\left(k_{F}+\delta k\right)$ : First Splin Flip. C) Electrons at the bottom of the subband $k=0$ flip their spins by jumping to the first empty state: Last Spin Flip. D) The fully polarized state.

ticle and is due to the failure of the constant interaction model especially for the dot. The condition in order to allow a spin flip is

$$
v_{F}\left(\omega_{c}\right) \delta k=g_{4}^{\perp}\left(\omega_{c}\right)-g_{4}^{\|}\left(\omega_{c}\right) .
$$

We explain in details the case of the electrons near the Fermi surface and the opposite one of the electrons in the bottom of the subband at $k=0$, as we show in Fig.(7).

The two electrons at the Fermi surface can flip their spins only by jumping from the $k_{F}= \pm N \delta k / 4$ doubly occupied to the nearest empty levels $\pm\left(k_{F}+\delta k\right)$. This transition is energetically provided if the growth in kinetic energy $2 v_{F} \delta k$ is equal to the reduction in interaction energy with one half of the remaining $(N-2)$ having spin up and the other half with spin down $\left(2\left(g_{4}^{\perp}\left(\omega_{c}\right)-g_{4}^{\|}\left(\omega_{c}\right)\right)\right)$. In the same way we can discuss what happens for two electrons which jump from the bottom of the subband $k=0$ to the first empty state, which now has all the levels singly occupied, so that the difference in kinetic energy is $2 v_{F}\left(\frac{N}{2}-1\right) \delta k$, while the gain in interaction energy is given by the spin flip $(N-2)\left(g_{4}^{\perp}\left(\omega_{c}\right)-g_{4}^{\|}\left(\omega_{c}\right)\right)$. Thus, we 


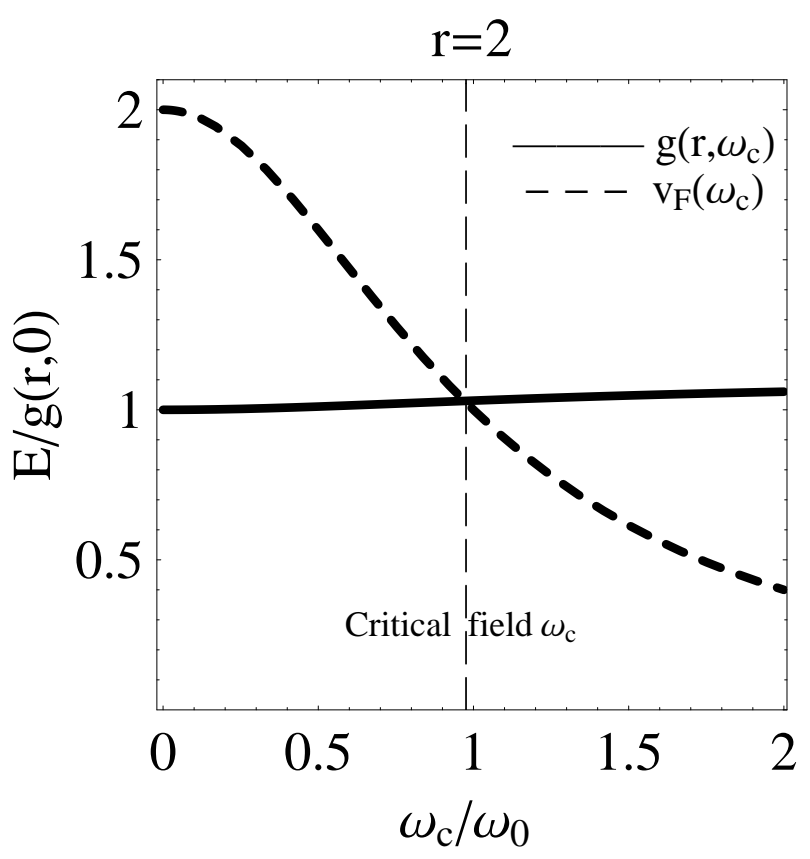

FIG. 8: Graphic calculation of the critical field for a long range interaction: the spin transition is due to the simultaneous reduction of the Fermi velocity and increasing of the electron electron repulsion.

can conclude that all the spins flip at the same critical value of the magnetic field, provided we can consider the interaction as a constant.

This very simple explanation fails if we assume non linear subbands or a long range interaction. In a future article, we will discuss this mechanism in more detail by taking in account also the long range interaction. Here we just want to suggest that the spin polarization takes place and it has some effects on the interaction parameter: $g_{i}^{\perp}$ disappears and the Fermi wavevector doubles yielding a further reduction of $g_{1}$ and $g_{2}$. We limit ourselves to show how the strong magnetic field has the effect of reducing the Fermi velocity and increasing the electron electron repulsion until a critical phenomenon (spin transition) occurs. In Figs.(1) and (8) we show the critical field from the crossing between kinetic and repulsion energy. We can conclude that a short range of interaction gives a lower critical field than a long range interaction. In the figure we just show the dependence of the critical field on the magnetic field and on the range of the interaction. In Fig.(9) we report the different regimes in the magnetic field-number of electrons plane, showing the various behaviours of the electron system in the wire when the magnetic field increases. This diagram is done in analogy with the ones obtained experimentally and theoretically for large Quantum Dots.

In a future article we will also discuss in some details the chiral Luttinger liquid in a ferromagnetic state analogous to a spinless system. There, it could be interesting to analyze the spin and charge excitations, in analogy

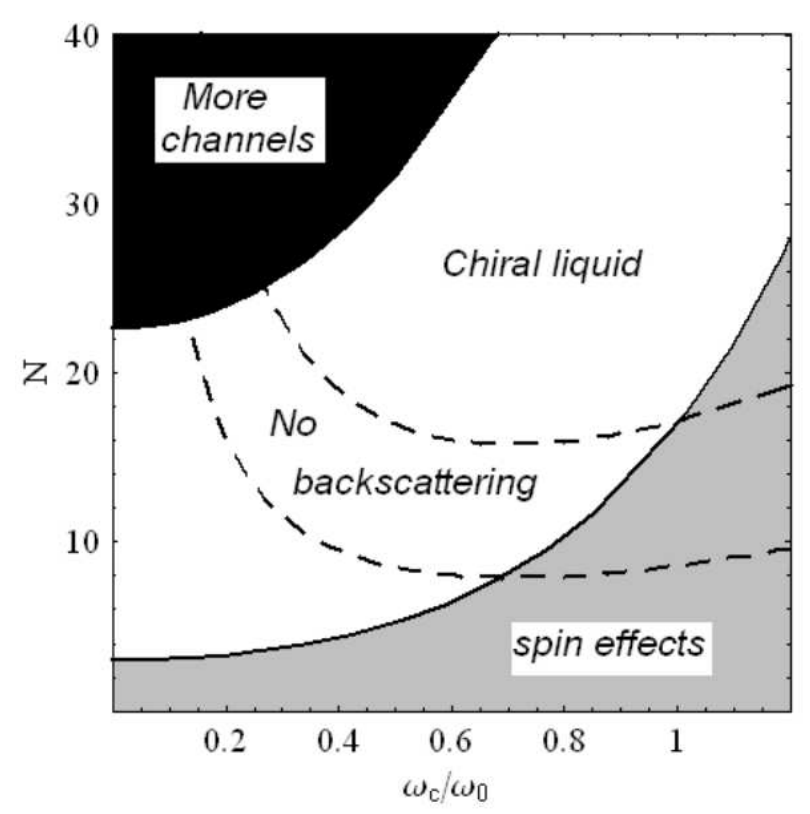

FIG. 9: A qualitative phase diagram in the plane magnetic field-number of electrons. We show that increasing the magnetic field we have the standard case, the backscattering suppression zone, the chiral liquid and the spin polarization. The Number of electrons in the vertical axis in this case is a free choice of the authors, in general it has to be coherent with the $L$ longitudinal dimension of the wire.

with the Quantum Dots and the Quantum Hall Ferromagnets.

\section{CONCLUSIONS}

In this work we have analyzed some properties of a Quantum Wire when a strong magnetic field is present. Most of the effects of the magnetic field are due to the rescaling of the electron electron interaction and the Fermi velocity. While the Fermi velocity always decreases with the magnetic field, the repulsive interaction between electrons suffers the competitive actions of the edge state localization and the characteristic length reduction.

We have described how the growth of a transverse magnetic field modifies the transport properties of a Quantum Wire under the hypothesis that the latter behaves as a 1 dimensional electron system. In this case, i.e. the one of Luttinger liquids, the tunneling transport properties are due to the large value of the critical exponent $\alpha$.

At a low magnetic field the usual Luttinger liquid behavior is predicted with some effects due to backscattering. When the magnetic field increases there is a strong reduction of backscattering, while for very high fields also forward scattering between opposite branches vanishes and a chiral Luttinger liquid appears. During 
the growth of the magnetic field the critical exponent is strongly reduced. A further rise of the field can cause the spin polarization which takes place as in a large QD, i.e. it does not depend on Zeeman or spin orbit effects but is due to the combined effect of the interaction and the magnetic field.

We also have discussed how the presence of one impurity can affect the conductance in the wire. The backward scattering reduction and the rescaling of the electron electron interaction could favor the weak potential limit (strong tunneling) by raising the temperature at which the wire becomes a perfect insulator $(G=0)$.

In the future we wish to analyze further the possible extension of this formalism to the study of Carbon Nanotubes and discuss with more detail the properties of a Luttinger liquid in a fully polarized state.

\section{Acknowledgments}

This work is partly supported by the Italian Research Ministry MIUR, National Interest Program under grant COFIN 2002022534.

\section{APPENDIX A: COMPARISON BETWEEN COULOMB INTERACTION AND MODEL}

In this appendix we discuss the difference between the Fourier transforms of the Coulomb interaction and of the model (eq.(11)).

We point out that the potential in the form of eq. (11) allows exact integration, in order to obtain the Fourier transform, and it regularizes the divergence appearing in the Coulomb potential. This function could be optimized varying the range parameter $r$, so that it could be quite similar to the Coulomb potential. The function $g\left(\delta k, \omega_{c}\right)$ is plotted for a very short range $(r=0.1)$ and for various values of magnetic field in Fig.(10).

The Coulomb potential is not so easy to integrate: we give its transform obtained by a numerical integration with a sort of regularization near the divergence, and normalized with respect to the value obtained at zero magnetic field $(g(0,0)=1)$. Also in this case we show in Fig.(10) the Fourier transform for various values of magnetic field.

Now we can conclude that the model fits well the Coulomb interaction, if we choose a short range parameter: Fig.(10) shows this good agreement.

We can conclude that a magnetic field in the limit of short range gives

$$
g\left(0, \omega_{c}\right) \approx g(0,0) \sqrt{\frac{\omega_{T}}{\omega_{0}}}
$$

so that we have the strongest interaction between electrons with quite similar momentum. From Fig.(10.a) and
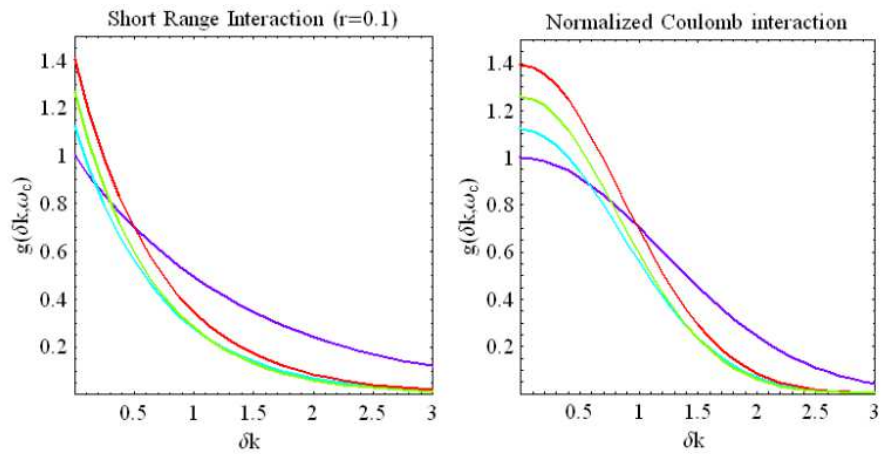

FIG. 10: a)On the left, the Fourier transform for various values of magnetic field of the model of interaction with a short range (0.1). b)On the right, the Coulomb potential case obtained with a numerical calculation and normalized with respect to the value obtained at zero magnetic field $(g(0,0)=1)$. A comparison shows a good agreement and an analogous magnetic field dependence.

Fig.(10.b) we can also argue that the function $V(\delta k)$ decays very rapidly when $\delta k$ increases for high magnetic fields.
1 T. J. Thornton, Rep. Prog. Phys. 58, 311 (1995).

2 C. W. J. Beenakker and H. van Houten Quantum transport in Semiconductor Nanostructures, vol. 44 of Solid state physics (Academic Press, New York, 1991).

3 R. Landauer, IBM J. Res. Dev. 1, 223 (1957).

${ }^{4}$ M. Büttiker, IBM J. Res. Dev. 32, 317 (1988).

5 C. L. Kane and M. P. A. Fisher, Phys. Rev. B 46, 15233 (1992)

6 A. V. Moroz, K. V. Samokhin and C. H. W. Barnes, Phys. Rev. Lett. 84, 4164 (2000).

7 A. V. Moroz and C. H. W. Barnes, Phys. Rev. B 61, R2464 (2000).

8 A. De Martino and R. Egger, Europhys. Lett. 56, 570 (2001).
9 B. J. van Wees, H. van Houten, C. W. J. Beenakker, J. G. Williamson, L. P. Kouwenhoven, D. van der Marel and C. T. Foxon,, Phys. Rev. Lett. 60, 848 (1988).

10 D. A. Wharam, T. J. Thornton, R. Newbury, M. Pepper, H. Ahmed, J. E. F. Frost, D. G. Hasko, D. C. Peacock, D. A. Ritchie and G. A. C. Jones, J. Phys.: Condens. Matter 21, L209 (1988).

11 L. I. Glazman, G. B. Lesovik, D. E. Khmel'nitskii and R. I. Shekhter, Pis'ma Zh. Eksp. Teor. Fiz. 48, 218 (1988); L. I. Glazman and M. Jonson, Phys. Rev. B 41, 10686 (1990).

12 S. Tomonaga, Prog. Theor. Phys. 5, 544 (1950); J. M. Luttinger, J. Math. Phys. 4, 1154 (1963); D. C. Mattis and E. H. Lieb, J. Math. Phys. 6, 304 (1965).

13 For a review see J. Sólyom, Adv. Phys. 28, 201 (1979) 
and J. Voit, Rep. Prog. Phys. 57, 977 (1994).

14 A. H. MacDonald, Phys. Rev. Lett. 64, 220 (1990); X.-G. Wen, Phys. Rev. Lett. 64, 2206 (1990); Phys. Rev. B 41, 12838 (1990); Phys. Rev. B 43, 11025 (1991); Int. J. Mod. Phys. B 6, 1711 (1992); U. Zülicke and A. H. MacDonald, Phys. Rev. B 54, 16813 (1996).

15 A. Furusaki and N. Nagaosa, Phys. Rev. B 47, 4631 (1993).

16 S. J. Tans, M. H. Devoret, H. Dai, A. Thess, R. E. Smalley, L. J. Geerligs and C. Dekker, Nature 386, 474 (1997); M. Bockrath, D. H. Cobden, J. Lu, A. G. Rinzler, G. Andrew, R. E. Smalley, L. Balents and P. L. McEuen, Nature 397, 598 (1999); Z. Yao, H. W. J. Postma, L. Balents, and C. Dekker, Nature 402, 273 (1999).

17 R. Egger and A. O. Gogolin, Phys. Rev. Lett. 79, 5082 (1997); C. Kane, L. Balents and M. P. A. Fisher, ibid. 79, 5086 (1997).

18 S. Tarucha, T. Honda and T. Saku, Solid State Comm. 94, 413 (1995).

19 A. Yacoby, H. L. Stormer, N. S. Wingreen, L. N. Pfeiffer, K. W. Baldwin and K. W. West, Phys. Rev. Lett. 77, 4612 (1996); O. M. Auslaender, A. Yacoby, R. de Picciotto, K. W. Baldwin, L. N. Pfeiffer and K. W. West, Phys. Rev. Lett. 84, 1764 (2000); M. Rother, W. Wegscheider, R. A. Deutschmann, M. Bichler and G. Abstreiter, Physica E 6, 551 (2000).

20 C. L. Kane and M. P. A. Fisher, Phys. Rev. Lett. 68, 1220 (1992); C. L. Kane and M. P. A. Fisher, Phys. Rev. B 46, 7268 (1992)

21 E. I. Rashba, Fiz. Tverd. Tela (Leningrad) 2, 1224 (1960) [Sov. Phys. - Solid State 2, 1109 (1960)]; Yu. A. Bychkov and E. I. Rashba, Pis'ma Zh. Eksp. Teor. Fiz. 39, 66 (1984) [JETP Lett. 39, 78 (1984)].

22 M. Governale and U. Zülicke Phys. Rev. B 66, 073311 (2002).

23 S. Bellucci and P. Onorato, Phys. Rev. B 68, 245322 (2003).
24 W. Häusler, L. Kecke, and A. H. MacDonald, cond-mat/0108290

25 S. Tarucha, D. G. Austing, T. Honda, R. J. van der Hage and L. P. Kouwenhoven, Phys. Rev. Lett. 77, 3613 (1996); T.H. Oosterkamp, J. W. Janssen, L. P. Kouwenhoven, D. G. Austing, T. Honda and S. Tarucha, Phys. Rev. Lett. 82, 2931 (1999).

26 V. Marigliano Ramaglia, F. Ventriglia and G. P. Zucchelli, Phys. Rev. B 48, 2445 (1993).

27 S. Bellucci and J. González, Eur. Phys. J. B 18, 3 (2000); S. Bellucci, Path Integrals from peV to TeV, eds. R. Casalbuoni, et al. (World Scientific, Singapore, 1999) p.363, hep-th/9810181. S. Bellucci and J. González, Phys. Rev. B 64, 201106(R) (2001); S. Bellucci, J. González and P. Onorato, Nucl. Phys. B 663 [FS], 605 (2003); S. Bellucci, J. González and P. Onorato, Phys. Rev. B 69, 085404 (2004); S. Bellucci and P. Onorato, Phys. Rev. B 71, 075418 (2005), cond-mat/0501051 S. Bellucci, J. González, P. Onorato, cond-mat/0501201

28 V. Meden, W. Metzner, U. Schollwock and K. Schonhammer, Phys. Rev. B 65, 045318 (2002); V. Meden, S. Andergassen, W. Metzner, U. Schollwock and K. Schonhammer, Europhys. Lett. 64, 769 (2003).

29 H. J. Schulz, cond-mat/9503150

${ }^{30}$ O. Klein, C. de C. Chamon, D. Tang, D. M. AbuschMagder, X.-G. Wen, M. A. Kastner and S. J. Wind, Phys. Rev. Lett. 74, 785 (1995).

31 O. Klein, D. Goldhaber-Gordon, C. de C. Chamon and M. A. Kastner, Phys. Rev. B 53, 4221 (1996).

32 O. Klein et al., in Quantum Transport in Semiconductor Submicron Structures, ed. Kramer (1996).

33 This phenomenon was observed in a transverse conductance measure where the peaks in the conductance correspond to the chemical potential of the N-electron system. 